DOI: https://doi.org/10.23925/ddem.i3.56272

Licença Creative Commons Atribuição 4.0 Internacional

\title{
OBJECIONES AL SISTEMA INTERAMERICANO DE PROTECCIÓN DE DERECHOS HUMANOS ${ }^{12}$
}

\author{
OBJECTIONS TO THE INTER-AMERICAN SYSTEM FOR THE PROTECTION OF \\ HUMAN RIGHTS
}

Jorge Isaac Torres Manrique ${ }^{3}$

\begin{abstract}
RESUMEM
El advenimiento de la vigencia del control o examen de la convencionalidad, así como, su inicial y paulatino acatamiento por los Estados parte, trajo consigo diversas resistencias, controversias y sobre todo expectativas, acerca de una supuesta naturaleza de panacea o suerte de sinónimo con la tan histórica como esquiva justicia.

En la presente entrega, el autor lleva a cabo una evaluación del desempeño de los organismos tutelares del Sistema Interamericano de Derechos Humanos (SIDH), principalmente el de la Corte Interamericana de Derechos Humanos (Corte IDH).
\end{abstract}

Palabras clave: convencionalidad; derechos humanos; Corte IDH; derechos fundamentales.

\begin{abstract}
The advent of the control or examination of conventionality, as well as its initial and gradual compliance by the States parties, brought with it various resistances, controversies and, above all, expectations, about a supposed nature of panacea or kind of synonym with the as historic as it eludes justice.
\end{abstract}

\footnotetext{
${ }^{1}$ El presente trabajo fue materia de exposición del autor en el Tercer Congreso Internacional de Derecho Convencional (México- 2021), organizado por el Colegio de Abogados Procesalistas Latinoamericanos (México), Asociación Mundial de Justicia Constitucional (Colombia), Universidad Autónoma de Chiapas (México) y Revista Jurídica Primera Instancia (México).

${ }^{2}$ Artigo escolhido e aprovado pelo Conselho Editorial Revista DD\&EM.

${ }^{3}$ Consultor jurídico. Abogado por la UCSM (Arequipa). Doctorados en Derecho y Administración, por la UNFV (Lima). Presidente de la Escuela Interdisciplinar de Derechos Fundamentales Praeeminentia Iustitia (Perú). Pesquisador Internacional del Grupo de Responsabilidade Civil e Processo Ambiental de la Escola Superior Dom Helder Câmara (Brasil). Colaborador Extranjero del Grupo de Investigação de Investigação Metamorfose Jurídica y Colaborador do Projeto de Pesquisa Constitucionalismo e Meio Ambiente: Sustentabilidade, Direitos Fundamentais e o Socioambientalismo na Sociedade Consumocentrista; ambos vinculados ao Programa de PósGraduação em Direito da Universidade de Caixas de Sul (Brasil). Miembro del Comité Editorial de la EDUCS Editora da Universidade de Caxias do Sul (Brasil). Miembro de la International Association of Constitutional LawIACL (Serbia). Miembro de la Asociación Mundial de Justicia Constitucional (Colombia). Miembro del Comité Científico Internacional del Instituto Jurídico Internacional de Torino (Italia). Miembro extranjero adjunto de la Asociación Argentina de Justicia Constitucional (Argentina). Miembro Senior de la Asociación Colombiana de Derecho Procesal Constitucional (Colombia). Miembro del Instituto Vasco de Derecho Procesal (País Vasco). Par Académico Evaluador de las firmas editoras: Corporación de Estudios y Publicaciones (Ecuador) y Ediciones Jurídicas de Santiago (Chile). Autor y coautor de diversos libros y tratados en Derecho Constitucional, Penal, Administrativo. CoDirector de los Códigos Penales Comentados de Ecuador, Colombia. CoDirector de los Tratados: Lavado de Activos, Litigación Oral Estratégica, Derecho Probatorio, entre otros. kimblellmen@outlook.com; http://lattes.cnpq.br/0707774284068716. http://orcid.org/0000-0001-5202-3886.
}

Revista Direitos Democráticos \& Estado Moderno |Faculdade de Direito da PUC-SP https://revistas.pucsp.br/index.php/DDEM | Nº. 03 | p.03-12 | Jul./Dez. 2021 
In this installment, the author carries out an evaluation of the performance of the guardianship agencies of the Inter-American Human Rights System (SIDH), mainly that of the InterAmerican Court of Human Rights (Inter-American Court of Human Rights).

Keywords: conventionality; human rights; Court IDH; Fundamental rights.

\section{PLANTEAMIENTO DEL PROBLEMA}

Acerca del mismo, es preciso dejar constancia de dos puntos neurálgicos a abordarse. En primer lugar, los excesivos tiempos que toma la Corte IDH para resolver los casos bajo su jurisdicción, así como, para ejecutar las mismas, en los términos dispuestos por la misma.

Ello, en el marco de la paradoja que se presenta entre la quintaesencia de la SIDH, CIDH y Corte IDH, para controlar y asegurar la salvaguarda de los derechos fundamentales y el objetivo desempeño de sus funciones, que va en contra de la propia naturaleza de la que fueron concebidos y puestos en vigencia.

\section{NATURALEZA DEL SIDH}

El sistema interamericano de protección de Derechos Humanos constituye un sistema internacional acordado por los Estados del sistema interamericano con el objeto de establecer estándares mínimos comunes en materia de respeto, garantía y adecuación de los ordenamientos jurídicos nacionales fijados convencionalmente en la Convención Americana sobre Derechos Humanos. Los Estados-Parte asimismo han acordado el establecimiento de un sistema de control del cumplimiento de dichos estándares por medio de dos organismos, la Comisión Interamericana de Derechos Humanos y la Corte Interamericana de Derechos Humanos. Esta última constituye una jurisdicción vinculante para los Estados Parte, cuyas sentencias constituyen obligaciones de resultado, las cuales no son objeto de recurso alguno. Dicha jurisdicción mantiene la supervigilancia del cumplimiento de los fallos hasta que el respectivo Estado Parte cumple con todas las medidas de reparación determinadas por la Corte. Éste es el objeto y fin del sistema interamericano de derechos humanos. ${ }^{4}$

\footnotetext{
${ }^{4}$ NOGUEIRA ALCALÁ. Humberto. El control de convencionalidad y el diálogo interjurisdiccional entre tribunales nacionales y corte interamericana de derechos humanos. En Revista de derecho constitucional europeo. Núm. 19. En línea: recuperado en fecha 9/10/21 de http://www.ugr.es/ redce/REDCE19/articulos/08_NOGUEIRA.htm. Chile, 2013. pp. 221- 222.
} 
Por otro lado, si en el Derecho Internacional clásico está claro que los recursos internos deben haberse agotado antes de someter el caso a una instancia internacional, en el sistema interamericano la solución que se ha adoptado es diferente. Los artículos 46 y 47 señalan que, para que sea admitida una petición, es necesario que se hayan agotado los recursos de la jurisdicción doméstica. En consecuencia, el momento crítico es aquel en que se vaya a admitir la petición, y no la oportunidad en que ésta haya sido presentada. ${ }^{5}$

Al respecto, es de verse en el caso contra Perú, ${ }^{6}$ que el art. $46 \mathrm{~N}^{\circ} 1$, letra a, de la Convención sólo establece que, para que una petición o comunicación 'sea admitida' por la Comisión se requerirá que se hayan interpuesto y agotado los recursos de la jurisdicción doméstica; esta disposición no exige que tales recursos se hayan agotado antes de presentar la petición, sino que se hayan agotado antes de que la Comisión decida admitir dicha petición. Así lo ha entendido la Corte, en un caso en que el Estado objetó que la petición había sido recibida cuando aún estaba pendiente un proceso penal contra las supuestas víctimas; según la Corte, si bien la Comisión recibió la denuncia cuando el procedimiento se hallaba pendiente de resolución definitiva ante la justicia militar, la mera presentación de aquella no motivó que la Comisión iniciara el trámite del asunto; el Tribunal observó que el trámite comenzó varios meses después de la presentación de la denuncia, cuando ya existía sentencia definitiva del órgano de justicia militar de última instancia, y que fue entonces cuando la Comisión hizo saber al Perú la denuncia presentada, para que el Estado pudiera aducir en su defensa lo que creyese pertinente.

\section{GRADO DE JUSTICIA QUE OFRECE EL SIDH}

Aparentemente la naturaleza del SIDH presenta un nivel muy superior de protección o salvaguarda de los derechos fundamentales; digamos que equiparable a la definición de la tan ansiada justicia.

En principio, como señalamos al inicio de la presente entrega es de reiterar que el sistema interamericano de protección de Derechos Humanos constituye un sistema internacional acordado por los Estados del sistema interamericano con el objeto de establecer

\footnotetext{
${ }^{5}$ FAÚNDEZ LEDESMA, Héctor. El agotamiento de los recursos internos en el sistema interamericano de protección de los derechos humanos. En línea: Recuperado en fecha 9/10/21 de usmp.edu.pe/derecho/3ciclo/derechos_humanos/Biblioteca\%20virtual/El\%20agotamiento\%20de\%20los\%20recu rsos\%20internos\%20en\%20el\%20SIDH.pdf. Lima, 2007, p. 43.

${ }^{6}$ CORTE INTERAMERICANA DE DERECHOS HUMANOS, CASO CASTILLO PETRUZZI Y OTROS, EXCEPCIONES PRELIMINARES, sentencia del 4 de septiembre de 1998, párrafos 52, 54, y 55.
} 
estándares mínimos comunes en materia de respeto, garantía y adecuación de los ordenamientos jurídicos nacionales fijados convencionalmente en la Convención Americana sobre Derechos Humanos. $^{7}$

Pero, a efectos de poder entender el grado de evolución de los ordenamientos jurídicos, es preciso desarrollarla, a efectos de también demostrar el avance paulatino de la configuración de la justicia propiamente dicha.

Luego cabe señalar que si en su momento, ante la llegada del Estado Constitucional de derecho (que dejó atrás al otrora Estado de derecho), que trajo también consigo la constitucionalización del derecho, en tanto que la Constitución Política se enarbolaba como tutela y garante de un nuevo orden jurídico; ahora nos encontramos asistiendo al ingreso a una nueva etapa, en la cual, es la Constitución que se torna complementada, viéndose la misma convencionalizada, dando lugar a la convencionalización del derecho. Así, esta actual etapa es en la que la Convención Americana sobre Derechos Humanos se yergue (si se quiere) por encima de las Constituciones de los Estados democráticos del mundo entero. Esto es, al Estado Convencional de derecho.

En ese sentido ${ }^{8}$, consideramos que dentro de los estadios o etapas de la escala evolutiva que comporta la administración de justicia, el Estado Convencional de derecho se ubica en la cuarta. Así, sostenemos como tales a: i) Estado de naturaleza, ii) Estado de derecho, iii) Estado constitucional de derecho, iv) Estado convencional de derecho, v) Estado restaurador de derecho y vi) Estado de Justicia.

La primera, el Estado de naturaleza, también denominada venganza privada, justicia privada o salvaje (justicia por mano propia, ojo por ojo...), aquella caracterizada por la justicia hecha por propia mano del afectado.

La segunda, vendría a ser el Estado de derecho, en el cual, es el derecho (y no las personas) quien toma las riendas de la administración y organización del poder. Específicamente, es la Ley quien tiene el respectivo protagonismo.

Estimamos que la tercera, el Estado constitucional de derecho, es aquella donde ya no manda la Ley, sino, la Constitución, generando que todo el aparato normativo de un Estado, se alinee o registre ineludible sintonía con lo preceptuado en la Constitución Política. Se le

\footnotetext{
${ }^{7}$ NOGUEIRA ALCALÁ. Humberto. Ob. Cit.

8 TORRES MANRIQUE, Jorge Isaac. Breves consideraciones a propósito del examen de la convencionalidad. Luces, sombras y agenda pendiente. En: Derecho Procesal Constitucional. Garantía jurisdiccional de la Constitución. VELANDIA CANOSA, Eduardo Andrés (Director Científico). Universidad La Gran Colombia. Bogotá. 2018, pp. 456- 457.
} 
denomina: Estado Constitucional de derecho (en la que hicieron su aparición los Precedentes Vinculantes del Tribunal Constitucional). Dicho sea de paso, conviene reiterar que la presente, es la actualmente nos rige y nos encontramos.

Así también, señalamos que la cuarta, el Estado convencional de derecho, es la que empezamos a ingresar en el sistema jurídico peruano, donde es la convencionalidad lo que rige como máximo señero de ordenamiento jurídico, por encima de la Constitución Política.

Aunque, si se quiere en strictu sensu, experimentamos una suerte de tutti frutti como ordenamiento jurídico. Esto es, que nos encontramos en esa surte de fusión de Estado Constitucional de derecho (que lucha por su consolidación) y Estado Convencional de derecho (en los pininos de su conocimiento, difusión y aplicación).

A continuación, como la quinta, la denominada: Estado restaurador de justicia, es la que se caracteriza por fortalecer o humanizar los mandatos de la Constitución Política, esto es, a la luz de lo preceptuado por la Justicia Restaurativa. Es decir, lograr que la eventual vulneración de los derechos fundamentales se vea debidamente resarcida, restituida, reconstruida, restaurada. La presente fase, sería la que eventualmente en no poco tiempo, arribaríamos.

Señalamos que de manera uniforme, los preceptos de la justicia restaurativa se asumen y aplican, con una orientación solamente penal y procesal penal.

Sin embargo, sostenemos que la justicia restaurativa, en tanto que se encuentra en concordancia con los postulados contenidos en la Constitución Política y la Convención Americana sobre Derechos Humanos, conforme corresponde a un Estado Constitucional de Derecho; debe ser también de aplicación a la totalidad de ramas del derecho. Y aunque la misma se acerca mucho a la justicia (puesto que no consigue restaurar en su totalidad o como corresponde), no consigue abrazarla.

La justicia no puede ser equiparable a la justicia restaurativa, ya que a diferencia de esta última, la carga o necesidad emocional no se plasma, debido a que por ejemplo, si el ofensor le robó al ofendido diez cabezas de ganado, pues, solo será justo o de justicia, que el ofendido reciba del ofensor de manera oportuna, el número completo y calidad (características) de lo robado y en extremo alguno, unas disculpas que puedan hacer las veces de bálsamo reductor del número y naturaleza de lo robado. Que no se nos malentienda, ya que no estamos en contra, demonizamos, rebajamos o desmerecemos a la justicia restaurativa, solo la comparamos con la justicia y que en dicha empresa queda evidentemente en rezago de esta última.

Finalmente, la sexta etapa (que consideramos la definitiva), resultaría ser la denominada Estado de justicia. Esta etapa se encontraría abocada al aterrizaje total en la justicia propiamente 
dicha, la única que debe existir; puesto que es la que realmente logra plasmar el significado de la institución jurídica priora, esto es, la justicia, la misma que enseñó Justiniano: "La Justicia es la constante y perpetua voluntad de dar a cada uno su derecho". Entonces, en esta fase no habrá cabida para apelaciones infructuosas y erróneas, como: “justicia privada”, "justicia legal”, "justicia constitucional”, "justicia convencional” o "justicia restaurativa".

Y lo más importante, radica en que el justiciable reclama con toda la razón del mundo que se le otorgue o administre justicia propiamente dicha, tal y como corresponde a la mismísima denominación de Administración de Justicia. Desafortunado resulta por decir lo menos, afirmar que el Sistema de Administración de Justicia responda a lo que el justiciable espera, con respuestas identificadas con las meras: justicia privada, justicia legal, justicia constitucional, justicia convencional o justicia restaurativa.

A todo esto, no olvidemos que en el primer párrafo se hizo referencia a que el Sistema Interamericano de Protección de Derechos Humanos constituye un sistema internacional acordado por los Estados del sistema interamericano con el objeto de establecer estándares mínimos comunes en materia de respeto, garantía y adecuación de los ordenamientos jurídicos nacionales fijados convencionalmente en la Convención Americana sobre Derechos Humanos.

Entonces, queda claro que dichos estándares fijados para los Estados son solo mínimos y comunes en materia de respeto, garantía y adecuación de los ordenamientos jurídicos nacionales fijados convencionalmente en la Convención Americana sobre Derechos Humanos. Queda claro que el Derecho Convencional lo dice sin decir, esto es, que el mismo no deviene o alcanza a la justicia propiamente dicha.

Sostenemos, que la existencia de dichos estándares mínimos, parece indicar que marcan el inicio de una progresiva protección y salvaguarda de los derechos fundamentales, a efectos de en su momento, llegar a ser máximos o totales, tal y como debiera corresponder. Y que, además, el Control de la Convencionalidad (propio de un Estado Convencional de Derecho), se encuentra ubicado en un poco más allá de la mitad del camino o proceso evolutivo de la administración de justicia, esto es, en el cuarto estadío, de seis. Lo que entre paréntesis, nos lleva a otra reflexión. Si son mínimos los estándares de defensa y salvaguarda que abraza el Control de Convencionalidad, se entiende que lo propio que manejan los ordenamientos jurídicos internos de cada Estado, cierta como preocupantemente, se encuentran por debajo del dicho estándar mínimo.

A mayor abundamiento, es de mencionar que la Corte Interamericana de Derechos Humanos, maneja unos plazos exageradamente extensos para la expedición de sus Opiniones 
Consultivas, sentencias y resoluciones, que incluso pueden llegar a los veinte años. A los que hay sumarle unos diez años en promedio para que el Estado parte dé cumplimiento a lo dispuesto en las mismas. Y finalmente, adicionarle diez años por lo menos, para que el caso agote la jurisdicción interna, a efectos de quedar expedito a ser de conocimiento de la referida Corte.

Así es de verse, que la sumatoria de los años resulta ser muy preocupante, puesto que resultan ser cuarenta años en promedio para que las decisiones de la Corte, sean finalmente efectivizadas, materializadas. Ello, además de los derechos fundamentales vulnerados que la propia Corte y los Estados parte, se encargan de vulnerar en el transcurso de dicho plazo.

Por lo cual, esa es una razón adicional por la que el Control de la Convencionalidad, que forma parte del Derecho Convencional, no cumple los objetivos que plantea su quintaesencia, ya que, el remedio termina siendo tal vez, peor que la enfermedad (la convencionalidad ha planteado el: "que", de manera aceptable, pero, se ha extraviado en el: "como") y por ende también, no amerita en modo alguno, ser equiparado a la naturaleza que ostenta la justicia.

Ergo, en mérito a lo sustentado, queda demostrado que el Derecho Convencional no es la panacea unimismable a la justicia (puesto que se encuentra por debajo de incluso, la justicia restaurativa). Así, tampoco es la luz, el oráculo, lo último en línea o el Escuadrón Zombie. Y dicho sea de paso, sostenemos que lo único igualable o equiparable a la justicia, es el último estadio referido, nos referimos así, al Estado de Justicia.

Obviamente, faltaría no pocas centurias o tal vez milenios, para que el Estado de Justicia se haga una realidad. Sin embargo, ello no debe resultar ser óbice para que la totalidad de los actores del sistema de administración de justicia, decidan desde ya, asumir el reto y decisión en términos de justicia, en virtud al principio de legitimidad; pues, es lo que única, exclusivamente y con justo derecho merece y exige el justiciable.

\section{TIEMPO QUE TOMA LA CORTE IDH PARA EXPEDIR SUS SENTENCIAS}

No constituye un secreto reconocer que a la Corte IDH le puede tomar entre quince y veinte años en concluir un caso.

Es más, si damos una mirada al Sistema Interamericano de Derechos Humanos, tenemos que la $\mathrm{CIDH}$, reconoce el notorio como excesivo retraso para la resolución de casos. 
Así tenemos, el pilar de protección y defensa, que incluye el sistema de peticiones, casos, soluciones amistosas y medidas cautelares, es una herramienta fundamental para la CIDH y para todos los habitantes del hemisferio. Representa un orgullo para el continente americano, reconocido internacionalmente por su objetividad, seriedad, consistencia y calidad jurídica. Teniendo en cuenta esta centralidad, y el gran retraso procesal que se ha acumulado desde la década de los 90, la Comisión ha enfocado su prioridad en un programa de reducción del atraso procesal. Luego de un proceso de consultas que involucró a más de 500 personas y 300 entidades, la CIDH aprobó su Plan Estratégico 2017-2021 con 5 objetivos estratégicos. El refuerzo del sistema de peticiones, casos, soluciones amistosas y medidas cautelares se identificó como el primero de tales objetivos, y el primer programa del plan constituye el Programa Especial para el Atraso Procesal. ${ }^{9}$

\section{TIEMPO QUE TOMA LA CORTE IDH PARA EJECUTAR SUS SENTENCIAS}

El presente punto resulta más preocupante aun, el tema de la ejecución de las sentencias de la Corte IDH; en tanto que la materialización del derecho al derecho fundamental a la tutela judicial efectiva, vale decir de manera específica, a la materialización de la tutela judicial.

Ello, puesto que en no pocos casos los Estados signantes postergan por años el cumplimiento de las resoluciones definitivas de la Corte IDH.

Entonces, la Corte IDH precisa estar haciendo seguimientos para lograr que los Estados lleven a cabo lo dispuesto en sus sentencias.

Es de lamentar, que el tiempo empleado para la ejecución de las mismas puede llegar en promedio hasta diez años.

\section{ANÁLISIS}

Es de referir, que hemos determinado la existencia de cuatro puntos a considerar para el análisis respectivo. Así tenemos:

En primer lugar, el que la naturaleza de la existencia del SIDH y por ende, la Comisión IDH y la Corte IDH, no se constituyen en sinónimo de la palabra justicia.

En segundo término, el excesivo como injustificado tiempo que le toma a la Corte IDH.

\footnotetext{
9 COMISIÓN INTERAMERICANA DE DERECHOS HUMANOS. Informe anual 2020. En línea: recuperado en fecha 9/10/21 de https://www.oas.org/es/cidh/docs/anual/2020/capitulos/IA2020cap2-es.pdf. 2020. Pp. 2- 3.
} 
En tercer lugar, nuevamente el inexplicable prolongado tiempo que le toma a la Corte IDH ejecutar sus sentencias.

Finalmente, en cuarto lugar, el cuestionamiento de los puntos referidos a efectos de determinar la legitimidad de la existencia del SIDH.

\section{OBJECIONES}

Así tenemos, que un SIDH que largamente no logra su objetivo, no logra a su vez demostrar la necesidad o contundencia de su existencia.

Ello, en tanto que si tomamos en cuenta los diez años que se requiere para agotar la vía interna. Luego, los casi veinte años para expedir las sentencias. Y para concluir, adicionar diez años más para tentar la ejecución de las mismas.

Consecuentemente, lo señalado no justifica la existencia y vigencia del SIDH, pues, claramente el remedio se tornó en algo peor que la enfermedad.

\section{CONCLUSIONES}

El SIDH viene haciendo un mea culpa acerca de su abdicación a su quintaesencia y compromiso con la salvaguarda de los justiciables que ven avasallados sus derechos fundamentales por parte de los Estados signantes, puesto, que reconoce que existe un gran retraso acumulado desde aproximadamente treinta años y en consecuencia pone en marcha un plan estratégico.

Dicho retraso viene generando un grave daño a la configuración del SIDH y a la salvaguarda de los derechos fundamentales de las personas. Sin contar, que a ello se debe adicionar, que no es suficiente considerar la agilización de la atención de los casos atrasados, sino también, que las sentencias emitidas por la Corte IDH sean debidamente ejecutadas y con la mayor celeridad por los Estados parte.

Preocupa, además, en tanto que tuvo que pasar no pocas décadas para que se lleve a cabo dicho reconocimiento y la implantación de dicha estrategia; en tanto que, el SIDH tiene como razón de ser salvaguardar los derechos fundamentales que fueron vulnerados por los Estados parte.

En tal sentido, resulta muy preocupante que la propia CIDH se venga constituyendo en una continuidad de las referidas vulneraciones. Además, sin tomar en cuenta que quien recurre 
a la jurisdicción internacional (SIDH), tuvo que haber esperado diez años en promedio para agotar la vía interna.

\section{SUGERENCIAS}

La CIDH y en general el SIDH tienen que evaluar el muy pronto establecimiento e implementación de nuevas medidas conducentes a lograr una puesta al día, agilización de la atención de casos con un atraso inconcebible.

Así también, lo propio para garantizar que la ejecución de sus sentencias se lleve a cabo en los plazos establecidos y en los términos requeridos.

\section{REFERENCIAS}

Comisión Interamericana de Derechos Humanos. Informe anual 2020. En línea: recuperado en fecha 9/10/21 de https://www.oas.org/es/cidh/docs/anual/2020/capitulos/IA2020cap2es.pdf. 2020.

Corte Interamericana de Derechos Humanos, Caso Castillo Petruzzi y Otros, Excepciones Preliminares, sentencia del 4 de septiembre de 1998, párrafos 52, 54, y 55.

FAÚNDEZ LEDESMA, Héctor. El agotamiento de los recursos internos en el sistema interamericano de protección de los derechos humanos. En línea: Recuperado en fecha $9 / 10 / 21$ usmp.edu.pe/derecho/3ciclo/derechos_humanos/Biblioteca\%20virtual/El\%20agotamiento\%20 de\%20los\%20recursos\%20internos\%20en\%20el\%20SIDH.pdf. Lima, 2007.

NOGUEIRA ALCALÁ. Humberto. El control de convencionalidad y el diálogo interjurisdiccional entre tribunales nacionales y corte interamericana de derechos humanos. En Revista de derecho constitucional europeo. Núm. 19. En línea: recuperado en fecha 9/10/21 de http://www.ugr.es/ redce/REDCE19/articulos/08_NOGUEIRA.htm. Chile, 2013.

TORRES MANRIQUE, Jorge Isaac. Breves consideraciones a propósito del examen de la convencionalidad. Luces, sombras y agenda pendiente. En: Derecho Procesal Constitucional. Garantía jurisdiccional de la Constitución. VELANDIA CANOSA, Eduardo Andrés (Director Científico). Universidad La Gran Colombia. Bogotá. 2018. 